\title{
Giant mycotic abdominal aneurysm and massive vertebral erosion. Still not a tale from the past
}

\author{
Łukasz Hapka ${ }^{1}$, Grzegorz Halena ${ }^{2}$, Maciej Pytka ${ }^{3}$ \\ ${ }^{1}$ Department of General Surgery, Specialist Hospital in Chojnice, Poland \\ ${ }^{2}$ Clinic of Cardiac and Vascular Surgery, Division of Vascular Surgery, Medical University of Gdansk, Poland \\ ${ }^{3}$ Department of Health Sciences, Pomeranian Academy, Slupsk, Poland
}

A 67-year-old crewman was airlifted from a container ship at the Namibian coast after reporting sudden lumbar pain. A plain X-ray was performed locally (Fig. 1A) and low-resolution computed tomography angiography (CTA) revealed a large abdominal aortic aneurysm (AAA) with vertebral body destruction. After the patient had been sent home, high-resolution CTA confirmed a $10 \mathrm{~cm}$ AAA and complete destruction of L3/L4 vertebral bodies (Fig. 1B).

The patient was admitted to the Department of Vascular Surgery and scheduled for an endovascular repair of AAA. Preoperative workup included C-reactive protein, procalcitonin and blood cultures. All were negative and excluded potential aortic infection (usually caused by Staphylococcus, Streptococcus, Salmonella). A stentgraft was implanted (Gore Excluder, Flagstaff, AZ, USA) resulting in aneurysm exclusion (Fig. 1D).

No further erosion of vertebral bodies was observed during 6-year follow up, the aneurysm shrank to $70 \mathrm{~mm}$ (Fig. 1C). The neurosurgeon initially considered external stabilization of the spine, however as the pain had completely subsided after repair, the patient was scheduled for observation only.

A phenomenon called "chronic contained rupture" of the aorta can be explained by a limited rupture of the posterior aortic wall leading to a gradual growth of AAA and vertebral erosion. Historically massive destruction of vertebrae, sternum or ribs were attributed to mycotic aneurysms and tertiary syphilis. Nowadays, the term mycotic aneurysm is often used for saccular aneurysms despite the lack of actual mycotic or bacterial infection. The above described dangerous combination of a large AAA and vertebral erosion could have been easily diagnosed with an abdominal X-ray and ultrasound and yet had been overlooked for years despite complaints by the patient.

The possibility of AAA in the diagnosis of chronic back pain should be noted.

Conflict of interest: None declared

Address for correspondence: Łukasz Hapka, MD, Department of General Surgery, Specialist Hospital in Chojnice, ul. Leśna 10, 89-600 Chojnice, Poland, e-mail: hapka@gumed.edu.pl 
Cardiology Journal 2018, Vol. 25, No. 6

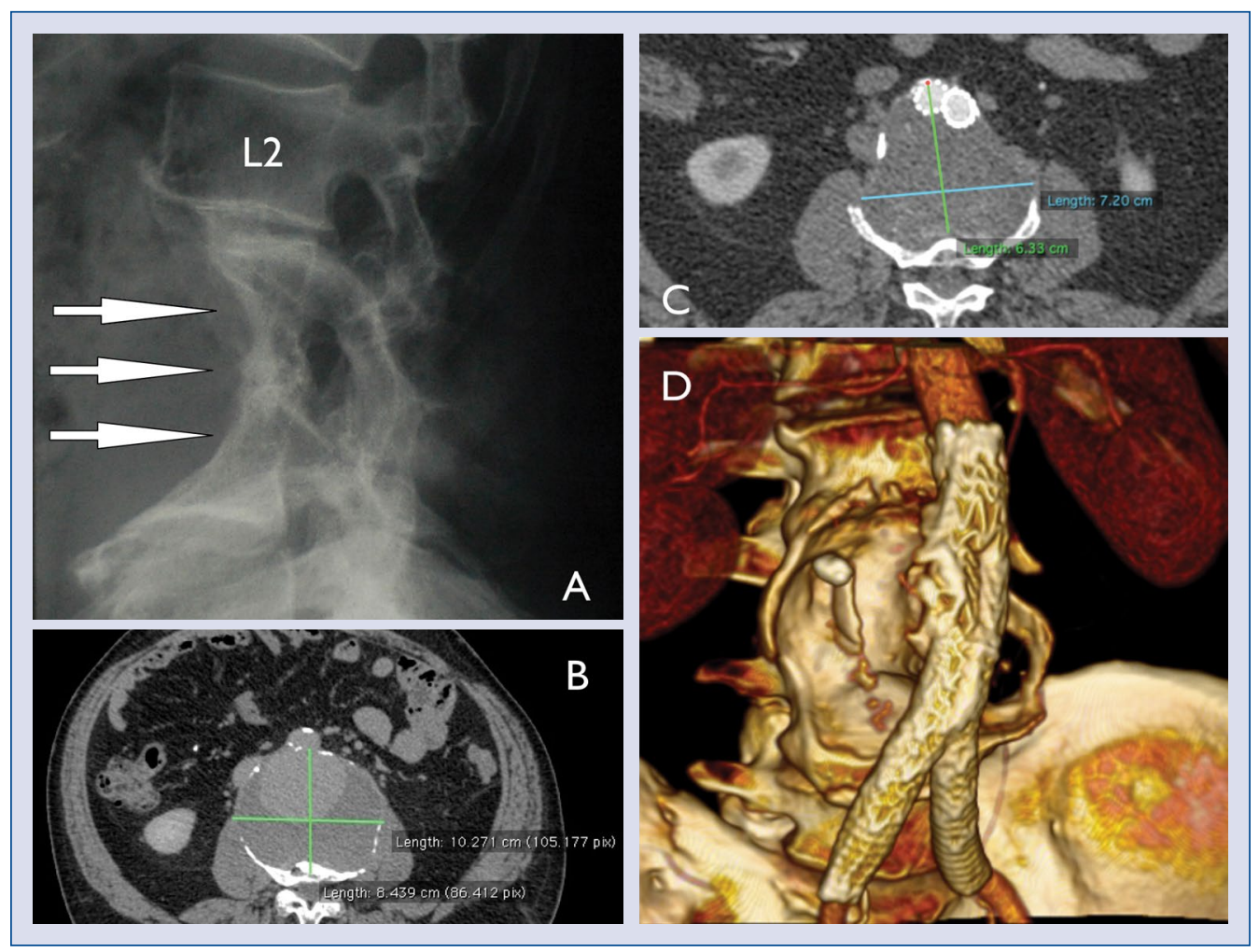

Figure 1. A. X-ray sagital view of the patient's the vertebral column; B. Preoperative reconstruction showing huge abdominal aortic aneurysm and almost complete destruction of a vertebral body; C. Postoperative reconstruction after exclusion of the aneurysm with stentgraft body; D. Postoperative three-dimensional reconstruction. 